\title{
PET/MR Imaging Consensus Paper: A Joint Paper by the Society of Nuclear Medicine and Molecular Imaging Technologist Section and the Section for Magnetic Resonance Technologists
}

\author{
C. David Gilmore, CNMT, RT(N), FSNMMI-TS, Chair ${ }^{1}$, Cindy R. Comeau, CNMT, RT(N)(MR), FSMRT, Co-Chair ${ }^{2}$, \\ Ann Marie Alessi, CNMT, NCT, RT(N) ${ }^{3}$, Maryann Blaine, MAT, RT(R)(MR ${ }^{4}$, Georges N. El Fakhri ${ }^{5}$, Jo Kathryn E. Hunt, \\ CNMT $^{6}$, David W. Jordan ${ }^{7}$, Brenda J. King, CNMT, FSNMMI-TS ${ }^{8}$, Anthony J. Sicignano, CNMT, ARRT(N) ${ }^{9}$, \\ Charles T. Stanley, RT(R)(CT)(MR) ${ }^{10}$, James T. Timpe, RT(N)(MR) ${ }^{11}$, and Nikki Wenzel-Lamb ${ }^{12}$ \\ ${ }^{1}$ Regis College, Weston, Massachusetts; ${ }^{2}$ Carnegie Hill Radiology, New York, New York; ${ }^{3}$ Biodex Medical Systems, Inc., Shirley, \\ New York; ${ }^{4}$ Brigham \& Women's Hospital, Boston, Massachusetts; ${ }^{5}$ Massachusetts General Hospital, Boston, Massachusetts; \\ ${ }^{6}$ Baptist College of Health Sciences, Memphis, Tennessee; ${ }^{7}$ University Hospitals Case Medical Center, Cleveland, Ohio; \\ ${ }^{8}$ B.J. King and Associates, LLC, Carson, California; ${ }^{9}$ Hospital of St. Raphael, New Haven, Connecticut; ${ }^{10}$ Stanford University School \\ of Medicine, Stanford, California; ${ }^{11}$ St. John's Hospital, Springfield, Illinois; and ${ }^{12}$ Society of Nuclear Medicine and Molecular \\ Imaging, Reston, Virginia
}

$\mathbf{H}$ ybrid imaging enters a new era with combining metabolic functional imaging as performed by PET and the superior anatomic imaging of MR, referred to as PET/MR. This new hybrid technology will play a significant role in enhancing the diagnostic capabilities in oncology and neurologic diseases. Two leading technologist professional organizations, the Society of Nuclear Medicine and Molecular Imaging Technologist Section (SNMMI-TS) and the Section for Magnetic Resonance Technologists (SMRT), have identified challenges for technologists in the merging of these 2 technologies.

The first meeting between the leadership of these 2 organizations took place in June 2011 in San Antonio, TX. Because of the unique imaging characteristics of these 2 modalities it was decided to form a SNMMI-TS/SMRT Task Force with representatives from each organization. This emerging hybrid technology is moving quickly into the diagnostic arena as both organizations have recognized the need for collaboration on establishing a consensus on certification and educational training requirements for technologists and radiographers entering this world of advancing technology.

\section{PURPOSE}

The purpose of this paper is to present unique modality issues as related to the operation of a PET/MR scanner, in

Received Mar. 27, 2013; accepted Mar. 27, 2013.

For correspondence or reprints contact: David Gilmore, Regis College.

E-mail: david.gilmore@regiscollege.edu

Published online Apr. 30, 2013.

COPYRIGHT (C) 2013 by the Society of Nuclear Medicine and Molecular Imaging, Inc.

DOI: 10.2967/jnmt.113.123869 the United States, as identified by the SNMMI-TS/SMRT Task Force as well as recommend specific regulations pertaining to certification and educational requirements for technologists to better prepare for the future.

\section{SNMMI-TS OVERVIEW}

Founded in 1954, the Society of Nuclear Medicine and Molecular Imaging (SNMMI) is a not-for-profit organization representing over 17,000 professionals specializing in the research and practice of nuclear medicine and working together to promote the science, technology, and practical application of the field. The SNMMI-TS, as part of SNMMI, is constantly striving to make a difference in the way nuclear medicine technologists receive academic and continuing education as well as information on new procedures, technologies, and equipment. Research has been a vital part of SNMMI-TS activities for many years, as has monitoring federal regulation and influencing decisions that affect the practice of nuclear medicine.

\section{SNMMI-TS Mission Statement}

SNMMI-TS is dedicated to the advancement of molecular and nuclear medicine technologists by providing education, advocating for the profession, and supporting research to achieve clinical excellence and optimal patient outcomes.

\section{SMRT OVERVIEW}

The SMRT of the International Society for Magnetic Resonance in Medicine (ISMRM) was established in 1991 and operates as a section of the ISMRM. The SMRT is the leading professional technologists' organization in the medical imaging community, providing education, professional advice, and support for MR technologists and radiographers globally. As an organization, the SMRT is committed to promoting communication and the dissemination of cutting-edge MR developments. The objective of the SMRT is to advance education and training, 
while striving to promote a high level of knowledge and professionalism among MR technologists and radiographers.

\section{SMRT Mission Statement}

- Enable MR technologists and radiographers throughout the world to achieve professional excellence through the provision of highquality education.

- Support and promote worldwide communication of current and emerging clinical and research information in the field of MR and provide a forum for its dissemination.

- Develop relevant and accessible member services and resources to enhance professional development.

- Provide informed advice and support to its global membership to enable the SMRT to work with local, regional, and federal agencies and organizations, to accomplish the above objectives.

\section{HISTORY OF HYBRID IMAGING}

Before 2000, PET images were fused with separately acquired CT images, with good registration success in the brain. This provided good simultaneous visualization of the anatomic detail from the $\mathrm{CT}$ and the physiologic detail of PET. Accurately fusing images in the body was more challenging, and good results were difficult to obtain. The PET/CT device that is currently in widespread use, combining a CT scanner and a PET scanner on a single concentric gantry, was introduced in 2000 and gained rapid acceptance because of the high spatial registration accuracy and the ability to use the rapidly acquired CT dataset for attenuation correction in place of transmission scans.

SPECT/CT has evolved along a pathway similar to that of PET/CT, although the need for attenuation correction is reduced in SPECT. However, SPECT/CT has not been as quickly accepted as PET/CT. In applications where visualization of the anatomy is needed, SPECT/CT has been readily adopted.

Separately acquired PET and MR images have been fused for display for some time. This process enables the benefits of PET/CT with the contrast sensitivity of MR. As with PET and CT fusion, registration of the datasets can be difficult and labor-intensive. Given the success of $\mathrm{PET} / \mathrm{CT}$, it has been desirable for some time to construct a successful combination device with a PET and MR scanner. The recent advances of avalanche photodiode technology have enabled the construction of a PET scanner that can operate in a strong magnetic field. Such devices can be constructed with a PET detector situated concentrically within the MR magnet. Improved active shielding of superconducting MR magnets has enabled "tandem" designs in which a conventional PET scanner can be located near an MR magnet and a patient can be imaged sequentially on each device by means of a common patient support couch. There are currently multiple medical equipment vendors marketing integrated PET/MR scanners that are approved by the U.S. Food and Drug Administration (FDA) for clinical use. Both concentric and tandem designs are available. Initial published reports indicate that the separate PET and MR performance of these devices is comparable to that of dedicated PET and MR scanners from the same manufacturers.

Given the recent development of new molecular imaging approaches such as breast-specific gamma imaging, dedicated cardiac SPECT systems, and positron-emission mammography, as well as the expansion of PET into cardiology using ${ }^{82} \mathrm{Rb}$-chloride and ${ }^{13} \mathrm{~N}$-ammonia, it is expected that PET/MR will find many new applications in organ- and region-specific imaging as well as the more familiar whole-body studies currently popular in PET/CT. Using dedicated and specific receiver coils, PET/MR can deliver superior contrast and detail sensitivity for localized examinations.

With improved hardware and software capabilities, MR has expanded into more advanced applications such as MR-guided focus ultrasound, and MR is being integrated into the surgical suite, thus becoming an invaluable tool in patient treatment management. Developments in the application of MR angiography include noncontrast imaging techniques that are widely used in routine examinations today.

\section{MARKET ANALYSIS}

At the time of writing, in spring 2012, there are currently 2 hybrid PET/MR machines available in the United States; the Siemens Healthcare Biograph mMR (approved by the FDA in June 2011) and the Philips Ingenuity TF System (approved by the FDA in November 2011). In addition, General Dynamics (GE) has a GE PET/CT + MR imaging machine that works to combine data only (not equipment).

Currently there are 2,085 sites performing PET imaging studies. Of the 2,085 sites, $300(14 \%)$ anticipate acquiring (through purchase or otherwise) PET systems over the next $3 \mathrm{y}$-inclusive of PET/CT, PET, and PET/MR. These data, from IMV PET Market Analysis 2011, show a significant decrease from the 2008 IMV PET Market Analysis, which indicated $22 \%$ of sites would be purchasing PET imaging systems (page 94). In addition, "if all sites that are currently planning to use PET/MR do so, $4 \%$ of the fixed PET/CT sites, $2 \%$ of the fixed PET sites, and $4 \%$ of the mobile users," will be using PET/MR technology by 2014 (IV-31) (IMV-2011 PET Imaging Market Summary Report).

A February 29, 2012, Diagnostic Imaging article (February 29,2012 ) reported that in a survey conducted in the summer of 2011 among readers, " 21 percent said they planned to purchase [PET/MR scanners] in the next six to 12 months, and 24 percent said that purchasing would depend on reimbursement. Another 16 percent planned to wait and see, while 38 percent had no purchase plans."

\section{EDUCATIONAL PROGRAM REQUIREMENTS: ENTRY-LEVEL}

\section{Entry-Level MR Technologists}

Entry-level MR imaging technologists must demonstrate competency-based American Society of Radiologic Technologists (ASRT) Performance Standards and American 
Registry of Radiologic Technologists (ARRT) Competency Requirements for MR imaging technologists. To perform at the expected competency level, the technologist should have appropriate didactic and clinical education in the following areas:

- Ethics and law as they apply to health care and the imaging sciences

- Patient care, assessment, education, and management

- Human anatomy and physiology and sectional anatomy

- Computers and informatics

- Medical terminology

- Pharmacology and drug administration

- MR imaging quality control and quality assurance

- Physical principles of MR imaging and instrumentation

- MR imaging procedures, parameters, and options

- MR pathology

- MR imaging safety

More specifically, an entry-level MR imaging technologist should be able to perform MR imaging procedures using established or accepted protocols to provide diagnostic images of:

- Brain and neck, including MR angiography of the head and neck

- Spine and thorax, including MR angiography of the great vessels

- Abdomen and pelvis

- Upper and lower extremities and joints

The technologist must also demonstrate appropriate patient identification and assessment skills, practice appropriate MR imaging safety procedures with regard to staff and patients, and provide necessary patient education and instructions. In addition, the technologist must demonstrate the ability to evaluate images on the basis of technical analysis, anatomy demonstrated, and overall image quality.

\section{Entry-Level Nuclear Medicine Technologists}

The entry-level nuclear medicine technology (NMT) curriculum is based on the accreditation standards required by the Joint Review Committee on Educational Programs in Nuclear Medicine Technology (JRCNMT) and the requirements set by the Nuclear Medicine Technology Certification Board (NMTCB) or the ARRT. The NMT professional curriculum includes the following didactic content areas:

- Patient care

- Cross-sectional anatomy

- Nuclear medicine statistics

- Nuclear medicine and radiation physics

- Radiation biology

- Radiation safety and protection

- Nuclear medicine instrumentation

- Quality control and quality assurance

- Computer applications

- Diagnostic nuclear medicine procedures

- Immunology

- Radiation therapy

- PET
- $\mathrm{CT}$

- Radionuclide chemistry and radiopharmacy

- Medical ethics and law

- Health care administration

- Health sciences research methods

- Medical informatics

- Pharmacology

The JRCNMT and the ARRT also indentify technical competencies in general areas requiring specific knowledge and skill sets in the following areas:

- Patient care

- Professionalism

- Radiation safety

- Instrumentation use and quality control

- Radiopharmaceuticals and pharmaceuticals

- Diagnostic procedures

- Radionuclide therapy

\section{Consensus Statement 1}

Although the nuclear medicine entry-level curriculum includes a limited amount of information on MR and, likewise, the MR entry-level curriculum includes a limited amount of information on nuclear medicine, both curricula are already filled to capacity. It is not in the best interest of either program, or the field, to add competencies to either curriculum at this time. It is recommended that the additional education needed for PET/MR technologists be in the form of advanced-level education.

\section{EDUCATIONAL PROGRAM REQUIREMENTS: ADVANCED LEVEL}

There appears to be creation of master's-level programs that could expand to include master's-level PET/MR.

\section{Consensus Statement 2}

Because of the complexity involved in operating PET/MR technology, for the safety of the technologist, the patients, and others involved, a new pathway for PET/MR imaging must be created. This should come in the form of an educational program that goes beyond entry-level for both modalities.

Prerequisites would include certification in at least one of the disciplines in this hybrid technology (NMT or MR) and ideally at least 2-3 y of clinical experience in that modality. The reasoning for this would be to increase the likelihood of appropriate patient care and critical thinking skills, as well as the understanding of the complexity of both modalities.

The intended advantage is that an advanced level of education for PET/MR technologists will allow one technologist to perform PET/MR imaging-this is a much more useful and practical solution to hybrid imaging. SMRT endorses the American College of Radiology (ACR) Guidance Document that states that at least $2 \mathrm{MR}$ imaging technologists should be present or at least $1 \mathrm{MR}$ imaging technologist and designated MR personnel (someone who is at least MR safety-trained) should be present. 
In addition, following the models currently in place for education, training, and certification of technologists in PET and MR individually:

1. A didactic training curriculum should be established containing specific elements of both PET and MR imaging. This should be available for technologists in several formats, including in-person classroom presentations, online distance learning, and self-paced written guides. This will ensure that adults with varying learning styles can benefit from this training. Much of this training is available within existing programs for MR imaging and PET and can be leveraged until a more permanent programmatic structure specific to PET/MR imaging can be established.

2. Clinical competencies in PET/MR imaging should be established to guide the clinical training and evaluation of the PET/MR imaging technologist, including a definition of appropriate qualifications for those supervising this training.

3. A registry/certification examination in PET/MR imaging technology should be developed, following a content specification containing appropriate elements drawn from existing PET and MR imaging certification examinations, in addition to new content specific to the unique nature of the combined modality.

Based on existing MR imaging content specifications and competency requirements (ARRT), the PET/MR imaging technologist should demonstrate full knowledge and proficiency in all aspects of general patient care (including MR imaging safety and radioisotope handling) and quality control. However, proficiency in head and neck, breast, cardiac, and whole-body MR imaging techniques is required by the initial applications of PET/MR imaging. Competency in the clinical use of spectroscopy and functional MR imaging techniques is also required.

Also, the entire current content outline of the NMTCB specialty examination in PET is appropriate for the PET/MR imaging technologist. Patient care, radiation safety, and safe handling and administration of radiopharmaceuticals are essential. Details of patient preparation and biodistribution, common PET radiopharmaceuticals, and quality control of PET scanners and hot lab equipment are also critical.

The PET/MR imaging technologist should receive education and training and demonstrate knowledge and competency in the areas of basic physics and instrumentation of both MR imaging and PET.

It is recommended that, to develop an advanced-level educational pathway for PET/MR technologists, a Dual Modality Content Specification survey and Gap Analysis for PET/MR imaging should be completed before the development of the curriculum.

\section{TRAINING CHALLENGES}

It is challenging at this time to fully specify the scope of education and training requirements that technologists will need to operate the PET/MR system since the field is still emerging. Education and training will have to include MR imaging education and safety requirements for the nuclear medicine/PET technologist and PET education and radiation exposure for the MR imaging technologist. The development of education is feasible and should be pursued and promoted.

It is not imperative that PET/MR imaging be permanently staffed by 2-technologist teams or by individuals who are fully PET- and MR imaging-certified. The development of programs and pathways specific to PET/MR imaging, drawing limited content from each area, is feasible and should be pursued and promoted.

\section{CERTIFICATION OF TECHNOLOGISTS}

There are currently 3 certifying organizations recognized by the SNMMI-TS and SMRT that certify nuclear medicine and MR technologists.

The ARRT is the world's largest credentialing organization for imaging professionals, with currently over 13,000 credentialed nuclear medicine technologists and over 28,000 credentialed MR technologists. However there are less than 1,000 that hold dual certification in both NMT and MR (statistic from e-mail from ARRT on March 15, 2012).

In addition, the NMTCB was formed for the purpose of creating and maintaining examinations for nuclear medicine technologists. The standards established by the NMTCB include educational requirements, practical experience, and successful completion of an appropriate competencybased examination. The NMTCB has registered 23,667 active certified nuclear medicine technologists (CNMTs). Of those, 642 also hold nuclear cardiology technologist (NCT) certification, 556 hold PET certification, and 58 hold both NCT and PET certification.

The Canadian Association of Medical Radiation Technologists (CAMRT) is Canada's national professional association and certifying body for medical radiation technologists and therapists across the country. There are 4 disciplines represented among CAMRT's membership: radiologic technology, MR, nuclear medicine, and radiation therapy. As the national certifying body, CAMRT develops and administers national certification examinations for the 4 disciplines among its membership, including nuclear medicine and MR.

The American Registry of MRI Technologists (ARMRIT) is an independent credentialing organization for MR. This organization accredits its own MR imaging educational programs and is a recognized certifying organization by the ACR.

\section{Consensus Statement 3}

As PET/MR continues to evolve and new applications are discovered, a new specialty certification examination should be explored to demonstrate competency in this new and complex hybrid imaging system. 
Pathway for Credentialing If MR Is First Specialty. Advanced educational program with minimum of 2 terms in addition to entry-level NMT program in MR imaging:

a) Radiation safety

b) Instrumentation use and quality control

c) Radiopharmaceuticals and pharmaceuticals

d) Diagnostic procedures

e) Radionuclide therapy

Pathway for Credentialing If NMT Is First Specialty. Advanced educational program with minimum of 2 terms in addition to entry-level NMT program in MR imaging:

a) Physical principles of MR imaging and MR imaging instrumentation

b) MR pharmacology and drug administration

c) MR imaging quality control and quality assurance

d) MR imaging procedures, parameters, and options

e) MR imaging pathology

f) MR imaging safety

\section{STATE LICENSURE}

Each state's current and future licensure may or may not include specific items, but we need to work with advocacy on showing value and licensing appropriate personal who are trained to operate PET/MR.

\section{Nuclear Medicine}

Licensure regulations for nuclear medicine technologists vary from state to state. The SNMMI-TS is currently working at a local level to establish uniform requirements for nuclear medicine licensure statewide. The Consumer Assurance of Radiologic Excellence (CARE) bill, if passed and enacted, will require the uniform regulations for personnel operating diagnostic equipment. Recommendations from the Task Force should be adopted concerning personnel qualifications.

\section{Medicare Improvements for Patients and Providers Act (MIPPA)}

In addition to the CARE bill, MIPPA, approved by the U.S. Congress in 2008, requires all nonhospital suppliers of the technical component of advanced diagnostic imaging services, nuclear medicine, MR, CT, and PET to obtain accreditation as a condition for reimbursement.

\section{Consensus Statement 4}

PET/MR technologists must be licensed in states in which nuclear medicine or MR licensure currently exists.

\section{CONTINUING EDUCATION REQUIREMENTS}

Each credentialing organization has continuing education requirements. Technologists must fulfill the requirements set forth by the certification board in which they received their certification.

\section{Consensus Statement 5}

It is the recommendation of the Task Force that the Advisory Board create a set number of credits in nuclear medicine/PET and MR imaging.

\section{STAFFING CHALLENGES}

Clearly, PET/MR imaging, much like previously introduced hybrid imaging technologies, will produce staffing challenges for diagnostic imaging departments. The challenge in staffing this new modality is coupled with the assurance of proper availability of staff to preserve both image quality and patient safety, while still maintaining the viability of this new hybrid imaging technology by avoiding overtaxing limited health care resources.

The goal of any staffing solution should be to create a safe environment with optimal patient care and image quality, but with the flexibility to provide the imaging technology in a variety of health care settings and physical spaces. The basis of safe staffing levels should come from current best practice and accreditation guidelines established for the individual modalities, with the highest recommended staffing levels of the two followed to maintain all aspects of quality patient care.

The ACR has written extensively on safe staffing guidelines for MR imaging environments, and its recommendations have been adopted as best practices by The Joint Commission. Therefore, any proper hybrid imaging staffing plan which involves MR imaging as a component of the imaging technology should include these recommendations. The ACR recommends that 2 staff members with proper MR imaging safety knowledge be present at all times in the scanning area during patient examinations. This does not mean that both technologists need to operate the equipment or that there cannot be 2 machines sharing one control room to satisfy these requirements. Because ACR has recommended 2 staff members, the consensus is that there will be 2 techs, a nuclear/PET-certified tech and an MR-certified tech.

\section{Consensus Statement 6}

SMRT endorses the ACR Guidance Document that states that at least $2 \mathrm{MR}$ imaging technologists should be present or at least $1 \mathrm{MR}$ imaging technologist and designated MR personnel (someone who is at least MR safety-trained) should be present.

Although not ideal, a hybrid approach is also acceptable during the transition period of training and certifying PET/ MR technologists-one certified PET technologist and one certified MR imaging technologist as long as both have training in radiation safety and MR imaging safety. This model would likely be more feasible with equipment using separate scanners sharing a common table. In this model, because each scan is essentially performed separately, each technologist could perform his or her portion of the combined examination. Likely, the equipment could also then be used individually by the appropriate technologist to perform either a stand-alone MR imaging examination or a standalone PET examination.

The ultimate goal for the future of the field is a PET/MR imaging technologist trained to be fully functional in both modalities. However, as outlined above, this will require more rigorous training, education, and certification beyond the hybrid-only requirements that might be established. 
Diagnostic imaging managers and directors will require the guidance and backing of both professional societies and accrediting bodies to enlighten the larger health care constituency regarding the need for both proper training and certification of technologists who perform these examinations, as well as the need to maintain proper staffing levels to ensure that we achieve the quality and safety necessary to provide acceptable patient care using PET/MR imaging.

\section{COLLABORATION AMONG MODALITY ORGANIZATIONS}

Over the past several years, with the emergence of new technology and, specifically, hybrid imaging, it has become increasingly clear that collaboration among experts, member organizations, and industry is essential to the continued growth and future of molecular imaging and improved patient care. In 2011, the SNMMI-TS and SMRT, understanding the importance and sophistication of this new PET/MR technology, began meeting to discuss possible and future collaboration opportunities. It was through these discussions that the SNMMI-TS and SMRT leadership determined that it was necessary to outline the basic principles of PET/MR.

Previously, within the imaging modality, there was no opportunity for the SNMMI-TS and SMRT to work together on a system that requires such vivid and unique areas of expertise-a CNMT and a certified MR technologist. Neither a nuclear medicine technologist (not certified in MR) nor an MR technologist (not certified in nuclear medicine) could operate a PET/MR machine properly and without potential undue harm to the patient. Therefore, it is only proper that the experts in the field, the members of the SMRT and SNMMI-TS, come together to identify an appropriate path forward to ensure the success of the PET/MR technologists within this new hybrid imaging era.

\section{CONCLUSION/SUMMARY AND NEXT STEPS}

PET/MR is being installed throughout the world. This document, approved in June 2012, is a first attempt between the SNMMI-TS and SMRT to create a recommendation and consensus for the technologist specialties to work together in the field to provide the best patient care possible.

\section{REFERENCES}

1. Kaplan DA. PET/MR: how feasible is implementation? Diagnostic Imaging Web site. http://www.diagnosticimaging.com/pet-mr/content/article/113619/2040136. Published February 29, 2012. Accessed April 5, 2013.

2. 2011 PET Imaging Market Summary Report. Des Plaines, IL: IMV; July 2011. 